\title{
Human cortical activity evoked by the assignment of authenticity when viewing works of art
}

\author{
Mengfei Huang $^{1+}{ }^{+}$Holly Bridge ${ }^{2 \dagger}$, Martin J. Kemp ${ }^{3}$ and Andrew J. Parker ${ }^{1 *}$ \\ ${ }^{1}$ Department of Physiology, Anatomy, and Genetics, University of Oxford, Oxford, UK \\ 2 Department of Clinical Neurology, FMRIB Centre, John Radcliffe Hospital, University of Oxford, Oxford, UK \\ ${ }^{3}$ Trinity College, University of Oxford, Oxford, UK
}

\section{Edited by:}

Idan Segev, The Hebrew University of Jerusalem, Israel

\section{Reviewed by:}

Nancy Zucker, Duke University

Medical Center, USA

Marian Berryhill, University of

Nevada, USA

\section{*Correspondence}

Andrew J. Parker, Department of Physiology, Anatomy, and Genetics, University of Oxford, Sherrington Building, Parks Road, Oxford OX1 3PT, UK.

e-mail: andrew.parker@dpag.ox.ac.uk

${ }^{\dagger}$ Mengfei Huang and Holly Bridge Joint first authors.
The expertise of others is a major social influence on our everyday decisions and actions. Many viewers of art, whether expert or naïve, are convinced that the full esthetic appreciation of an artwork depends upon the assurance that the work is genuine rather than fake. Rembrandt portraits provide an interesting image set for testing this idea, as there is a large number of them and recent scholarship has determined that quite a few fakes and copies exist. Use of this image set allowed us to separate the brain's response to images of genuine and fake pictures from the brain's response to external advice about the authenticity of the paintings. Using functional magnetic resonance imaging, viewing of artworks assigned as "copy," rather than "authentic," evoked stronger responses in frontopolar cortex (FPC), and right precuneus, regardless of whether the portrait was actually genuine. Advice about authenticity had no direct effect on the cortical visual areas responsive to the paintings, but there was a significant psycho-physiological interaction between the FPC and the lateral occipital area, which suggests that these visual areas may be modulated by FPC. We propose that the activation of brain networks rather than a single cortical area in this paradigm supports the art scholars' view that esthetic judgments are multi-faceted and multi-dimensional in nature.

Keywords: fMRI, visual perception, Rembrandt, social neuroscience, psychophysiological interaction

\section{INTRODUCTION}

Viewing art is popular, pleasurable, and for the most part an unsolved puzzle when it comes to the neurological mechanisms underpinning this experience. Much of the pioneering work on the neurology of art tended to focus on what has been called "neuro-esthetics" and has aspired to tell us why we find something beautiful. This broad-brush approach tells us little about the complex mechanisms that shape an individual viewer's response to a specific work of art within powerfully determined contexts. In particular, it is incapable of handling the intricate interactions of form and content within the framework of strong viewer expectations. If neuroscience is to speak effectively to art historians, it requires new questions and different methods. In this paper we are testing one such approach, as an early step in realigning the interaction of art history and neuroscience. The topic we have selected is that of how assertions of authenticity shape what we see.

Determination of the authenticity of artworks is of great importance in the art world: authenticity brings a scholarly value in shaping art historical understanding, has direct and potentially huge consequence for monetary value, and, most relevantly here, is presumed to have an impact on the individual viewer's experience. Declaring an artwork to be a forgery completely changes the reception of a picture by the viewer; suddenly, "we can see its every flaw" (Wynne, 2006).

Information about the authenticity of an artwork may therefore set a context for the perception of the art, with the capacity to modulate responses in the visual brain. The modulation of visual responses by contextual information is the focus of a great deal of current research. Selective attention (Kastner et al., 1999; Kastner and Ungerleider, 2000), reward (Shuler and Bear, 2006; Krawczyk et al., 2007), and working memory (Zaksas and Pasternak, 2006; Offen et al., 2009) are all examples of changes of context that set an appropriate bias on lower level responses within the brain. The viewing of artwork, particularly the pleasure or fascination it induces (Kawabata and Zeki, 2004, 2008), is also a multidimensional experience, extending beyond the purely visual. The way in which non-visual information about authenticity may alter the viewer's experience has been acknowledged in the context of art history and scholarship (Bossart, 1961; Goodman, 1976). Similar influences of non-sensory information occur in other sensory systems and in other culturally significant contexts (McClure et al., 2004).

When attempting to bridge the divide between neuroscience and studies of art, an initial step has been to summarize the multi-faceted experience of viewing art with a single rating of an artwork's esthetic impact (Kawabata and Zeki, 2004). Although such a generalization could form a useful starting point, scholars in the art world have found little of use in such an approach. By contrast, here we focus on a particular aspect of the viewer's experience, which art scholars generally agree is of significance, namely whether a work of art has been assigned as authentic or derivative. For less expert viewers, advice from experts is highly influential. Thus, there may be a strong effect of social influence on perception in this setting (Asch, 1956; Mojzisch and Krug, 2008). 
For this reason, we designed our study to concentrate upon a highly regarded artist (Rembrandt) and on a particular band of expertise about that artist among the participants. We sought participants who were familiar with the name of Rembrandt but were not expert or trained viewers of art. Studies of expert or trained viewers are of course potentially of great interest, but we concluded that the nature of expertise may be highly particular. For example, there are experts who devote themselves to the study of fakes. We reasoned that such experts might find the viewing of fakes more rewarding than the viewing of authentic works. Another example is that experts in modern art might find Rembrandt relatively unexciting and therefore such persons might not care much whether they are looking at a fake or authentic picture. On the other hand, a true Rembrandt expert would be able effortlessly to spot the discrepancies between our assignments of authenticity and the underlying reality as to whether the images are genuine or fake. All these comments emphasize that expertise, especially in its elaborated forms, is individual and particular. For this reason, we concentrated upon the experience of the average viewer in an art gallery, who will look at the artworks in the context of advice from experts (displayed on the gallery wall or in the exhibition guide) about the authenticity of the works.

Using functional magnetic resonance imaging (fMRI), we examined the brain's response to assignment of authenticity during the viewing of "Rembrandt" portraits. The assignment of authenticity did not always reflect the true status of the work: in reality, sometimes the viewed artwork was truly authentic and sometimes it was a copy. Rembrandt portraits are particularly suitable for our study because there is a large number of examples, both real and false, whose authenticity has been systematically determined, although still leaving some room for dispute. Hence we were able to determine the brain's response to contextual information generated by the assignment of authenticity, separately from the visual impact that is generated by a genuine or fake image.

The pattern of brain responses we identify here indicates that the major effects of assignment of authenticity are not directly visual, nor even unitary; rather, changes are observed in the interaction between multiple brain regions that all make relevant contributions to the viewer's experience.

\section{EXPERIMENTAL METHODS PARTICIPANTS}

Fourteen human participants (8M/6F, age 20-27, two left-handed) were recruited based on a screening questionnaire (see Appendix). The questionnaire ensured that all participants were amateur viewers (visit art museums $<5$ times/year; no extensive art training) and screened out individuals who dislike Rembrandt to avoid potentially confounding variables. Most importantly, it confirmed the participant's familiarity with Rembrandt, with all participants ranking him among the top 25 artists of all time, or higher. Establishing such esteem ensures that the difference between AUTHENTIC and COPY is salient.

All methods were conducted in accordance with NHS Ethics Reference 06/Q1604/86 and all participants provided written, informed consent. Each participant was provided with the following instructions before commencing the scanning session.
"In this experiment you will see a sequence of 50 Rembrandt paintings. Before each image appears, an audio prompt will announce whether the upcoming painting is 'authentic' or a 'copy' (Please see background for further information on copies). A blank screen will appear for a few seconds after each image to allow you to relax your gaze. A fixation cross will signal for you to focus again and an audio prompt will arrive shortly for the next image. Interspersed within the sequence will be three scrambled images. You will be told when to expect a scrambled image by the audio prompt 'neither."

The following background on Rembrandt and the definition of copies of artwork was provided for all participants to read.

Rembrandt is recognized as one of the greatest of all painters and etchers. Working in Amsterdam, he experienced great success in the first part of his career, while his latter years were dogged by financial problems (largely self-inflicted) and social difficulties. He was particularly famed for his portraits, which seem to present his sitters' personalities in a profound manner. No artist ever created such a range of self-portraits, which seem to comprise a kind of painted autobiography.

"Rembrandt van Rijn, 1606-1669

His early style was relatively detailed and naturalistic, but his way of handling paint became increasingly free. In his later works, in particular, thick, vigorous brush strokes, and large swathes of color evoke forms and textures rather than describing them in detail. The figures often emerge from dark or neutral backgrounds.

\section{What is a copy?}

Rembrandt taught a number of pupils who were adept at producing work in his various styles. He was much copied and imitated by painters other than pupils, both in his own lifetime and later. As prices for his works escalated, he also became the subject of forgery. What was actually Rembrandt and what was not by him became very confused. The Rembrandt Research Project in Holland was set up to sort out the confusion. In this experiment, works of art labeled as "COPY" refer to pieces that were produced by pupils, followers, or forgers."

The main idea behind the provision of this information was to reduce the potential for participants to bring potentially incorrect and confounding notions to this study. For example, there often a good deal of confusion in lay-usage about the word "fake," which is often presumed to mean some active attempt at forgery. Clarification on points such as these is likely to provide the various participants and sub-groups of the study with a consistent contextual setting prior to the main experiment.

At the end of each person's brain scan, a second questionnaire (see Appendix) was conducted to learn about the person's response to the Rembrandt images. Some of the responses to these questions were used to assist the interpretation of the outcome of the scanning experiments. This questionnaire also asked about whether the participants had fallen asleep during all or part of the scanning: none reported that they had. 


\section{SELECTION AND PRESENTATION OF VISUAL IMAGES}

Rembrandt's portrait works were prolific and much copied, creating a large set of both genuine and derivative works (Van Sonnenburg, 1995). We obtained a set of 50 high-resolution, color, digital reproductions from the University of Amsterdam Rembrandt catalog (Seinstra, 2010), carefully selected for a balance of male and female portraits, in addition to similar numbers of each pose (frontal, three-quarter pose to the right, and three-quarter pose to the left). All images were re-sized to fit the $1024 \times 768$ pixel projector screen placed $1210 \mathrm{~mm}$ from participant, which resulted in images that were 600 pixels in height $\left(\sim 20^{\circ}\right.$ of visual angle) and varied between 450 and 559 pixels in width. Within this set of 50 images, half of these (25) have been genuinely attributed to Rembrandt himself, while the other half are considered to be in the style of Rembrandt by someone else. Purely for convenience, we refer to the second of these groups as FAKE, even though not all of these works have been created as forgeries with intent to deceive.

Three portraits were scrambled, by converting each image into the Fourier domain, scrambling the phase of each frequency component, and then transforming back into image space (Prins, 2007). The scrambled portraits were interspersed at the beginning, middle, and end of the image sequence to provide a baseline for the cortical response to unstructured visual stimuli. Scrambled portraits were cued with the statement: "This is neither."

Participants were not given specific viewing instructions and were told to view each image as they pleased. Each participant viewed a randomly ordered sequence of 53 images, 25 of which were cued as AUTHENTIC, 25 cued as COPY, and three scrambled images cued as NEITHER. The participant heard a statement "This is authentic" or "This is a copy" - immediately prior to seeing an image of a Rembrandt or Rembrandt-like portrait (Figure 1). Audio recordings were used in place of visual text, to maintain identical visual stimulation between the two test conditions.

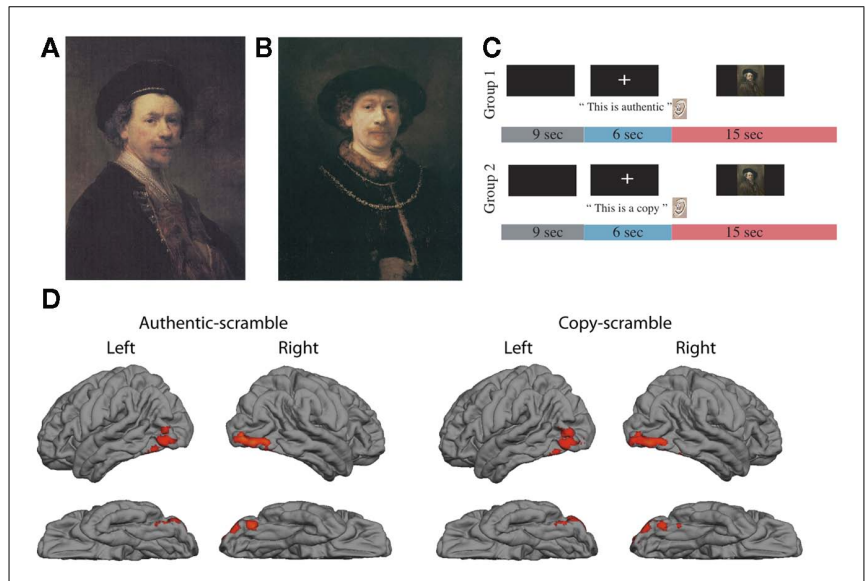

FIGURE 1 | (A) Sample of genuine Rembrandt portrait (REAL); (B) derivative portrait in the style of Rembrandt (FAKE); (C) sequence of auditory cues and image presentation; (D) brain activations in occipital and temporal cortex generated by presentation of portraits after subtraction of activations generated by scrambled images of portraits; data averaged across 14 participants, red regions show significant BOLD activations during period of image presentation ( $Z>2.3, p<0.05$, corrected for multiple comparisons).
A 15-s viewing time was chosen to balance the interest of maximizing the number of trials against the provision of adequate time for a participant to examine a painting as he/she would normally do, outside of an experimental setting. Average viewing time for a work of art is reported to be $27 \mathrm{~s}$ with a median of $17 \mathrm{~s}$ (Smith and Smith, 2001). With a 15 -s viewing/15 s rest paradigm, participants confirmed that they had adequate time to view each painting and take a break between trials.

Participants were divided into two groups. Group 1 (4M, 3F) viewed each painting under the opposite expectation as Group $2(4 \mathrm{M}, 3 \mathrm{~F})$. For example, the same painting would be cued as AUTHENTIC to Group 1, and as COPY to Group 2. The sequence of paintings was kept constant but presented in reverse order for half of the participants, to control for any effects of lapsing attention, as participants grew more tired toward the end of the experiment.

Out of the 25 paintings cued as AUTHENTIC, 13 were true, authenticated Rembrandts (REAL), and 12 were not (FAKE). Out of the 25 paintings cued as COPY, 13 were truly copies (FAKE), 12 were truly authentic (REAL). The relative numbers of REAL and FAKE works were reversed for the AUTHENTIC and COPY categories for Group 2.

From hereon, the first term in upper case refers to the actual identity of the painting, and second term refers the experimental expectation given each participant, such that a REALAUTHENTIC is both authentic in reality and cued as such; a FAKE-AUTHENTIC is a derivative work in reality, but is cued as AUTHENTIC. Omission of the first or second term signifies that the omitted variable is not specified, and both factors are included (e.g., AUTHENTIC includes both REAL-AUTHENTIC and FAKE-AUTHENTIC).

\section{fMRI DATA COLLECTION AND ANALYSIS}

Scanning was performed on a Siemens Trio 3T scanner with a 12-channel head coil at the Oxford Centre for Clinical Magnetic Resonance Imaging (OCMR). Each functional scan consisted of 530 volumes collected in $1590 \mathrm{~s}(\mathrm{TR}=3 \mathrm{~s}, \mathrm{TE}=30 \mathrm{~ms}$, voxel resolution $=2 \mathrm{~mm} \times 2 \mathrm{~mm} \times 2 \mathrm{~mm}$ ). Whole brain volumes were acquired to enable analysis of sensory and cognitive regions. We also acquired for each subject a high-resolution whole head T1anatomy scan (MPRAGE $1 \mathrm{~mm} \times 1 \mathrm{~mm} \times 1 \mathrm{~mm}$ voxels, 192 slices, $\mathrm{TR}=15 \mathrm{~ms}, \mathrm{TE}=6.0 \mathrm{~ms}$ ), optimized for gray- and white-matter separation. All analysis was carried out with the FMRIB Software Library (FSL; Smith et al., 2004; Woolrich et al., 2009).

Pre-statistical processing was applied as follows: skull and other non-brain voxels were removed using the brain extraction tool (BET; Jenkinson et al., 2002; Smith, 2002). Motion correction was applied with FMRIB's linear registration tool (MCFLIRT; Jenkinson et al., 2002). Data were spatially smoothed using a Gaussian kernel (full width at half maximum FWHM $=5 \mathrm{~mm}$ ). Highpass temporal filtering removed low frequency noise and slow drift. Each voxel's time series was divided by its mean image intensity and converted to a percent signal modulation and the time series of voxels within each restricted visual area mask was averaged. Statistical analysis on voxel time series was carried out using FILM (FMRIB's improved linear model) with local autocorrelation correction (Woolrich et al., 2001). Low-resolution functional 
data, high-resolution T1-anatomy and standard space templates were co-registered using FLIRT. $Z$-statistics were thresholded for individual voxels at $Z=2.3, p=0.01$, with the cluster significance for multiple comparisons correction set to $p=0.05$. Each participant's functional activity was registered to Montreal Neurological Institute (MNI) 152 standard space using FLIRT (Jenkinson and Smith, 2001). A general linear model (GLM) was applied to each participant's data using FMRIB's fMRI expert analysis tool (FEAT).

A single group average analysis was carried out with FEAT, which conducts a $t$-test independently for each voxel, and converts the resulting $t$-statistic to a $Z$-score thresholded at $2.3(p<0.01)$. Gaussian Random Field Theory was used to correct for multiple comparisons, the $Z$-statistic threshold was set to $Z=2.3$ with a cluster significance threshold of $p=0.05$.

Regions showing significant activation in these contrasts were further characterized by calculating the mean \%signal change over a given region of interest (ROI) for each participant using a FEATquery. Masks were created to define each ROI as a sphere centered upon the peak voxel in the group analysis. A 5-mm radius was chosen by convention for $2 \mathrm{~mm}$ voxel-size data. A $t$-test was applied to the group mean \%signal change and group SD. Note that the GLM analysis and the \%signal change both take into account the between-participant variance, but only GLM analysis also includes within-participant variance.

Resulting areas of activation were identified anatomically with the aid of the Harvard-Oxford Cortical Atlas and by comparing MNI coordinates to those found in previous literature. For visualization, brain activations were superimposed on the pial surface of the average brain created in Freesurfer.

A psycho-physiological interaction (PPI) analysis (Friston et al., 1997) investigates functional connectivity by looking for a difference in the correlation of activity between two areas during one psychological condition compared with another condition. Due to the interest in top-down modulation, the FPC, which differentially responded to AUTHENTIC vs. COPY (Figure 3), was chosen as the seed ROI. A right FPC mask thresholded at $Z=2$ was made from the group level analysis of COPY-AUTHENTIC. This standard space mask was then transformed back into the functional space of each participant using FLIRT (Jenkinson and Smith, 2001). Each participant's resulting mask was then used to identify the peak voxel that will serve as the functional ROI seed for that participant's PPI. Selecting each participant's seed individually accounts for anatomical heterogeneity across participants, and allows for reduced seed ROI size, thereby improving the signal.

Three explanatory variables were employed for the PPI; (1) the psychological regressor, corresponding to the COPYAUTHENTIC condition, (2) the physiological regressor, the timecourse of the seed ROI, and (3) the PPI regressor, the interaction between the psychological and physiological regressors. The PPI for each subject was then consolidated into a single average group analysis, following the same specifications as previously described.

Laterality indices were calculated using the LI-toolbox (Wilke and Lidzba, 2007) run in SPM8. A weighted-bootstrapping method of LI calculation was employed using the frontal lobe standard LI-toolbox template (Wilke and Schmithorst, 2006) during COPY trials relative to AUTHENTIC. The LI formula used was $\mathrm{LI}=(\mathrm{L}-\mathrm{R}) /(\mathrm{L}+\mathrm{R})$, therefore resulting in positive values for left and negative for right lateralization. Apart from the calculation of laterality index, all the main analyses reported in this paper were conducted with and without the inclusion of the left-handed subjects in the data analysis. No substantially different conclusions were reached.

\section{RESULTS}

\section{RESPONSE TO THE VISUAL CONTENT OF THE ARTWORK}

Figure 1 presents examples of a genuine Rembrandt portrait (Figure 1A, REAL) and a derivative or fake (Figure 1B, FAKE), as well as the presentation sequence for the auditory cue about authenticity (either AUTHENTIC or COPY) and the visual image (Figure 1C). To check for an appropriate response to the artworks, the activation to either AUTHENTIC or COPY relative to scrambled portraits was measured. The activity, pooled across the 14 participants, yielded a characteristic, three-blob chain, reflecting activity in lateral occipital complex (LOC), occipital fusiform gyrus, and temporal occipital fusiform cortex, based on HarvardOxford Cortical Atlas (Figure 1D, RED regions, $Z>2.3$, corrected for multiple comparisons). Both stimulus types elicited similar patterns of activity. The area on the fusiform gyrus selectively responds to images of faces (Kanwisher et al., 1997), and the LOC and temporal fusiform cortex have been found to be involved in object recognition (Grill-Spector et al., 1999; Ishai et al., 2000).

\section{ASSIGNMENT AS COPY OR AUTHENTIC GIVES SPECIFIC ACTIVATIONS IN NON-VISUAL AREAS}

The occipito-temporal areas of the cerebral cortex are known to be visual and these areas provided responses specific to the structure of the portrait images. Therefore, we also examined these areas to test whether they were differentially activated in response to assignment of authenticity, but nothing significant emerged. However, other brain regions, outside these areas, did have differences in response to assignment of authenticity.

Interestingly, the more distinct differences were in favor of greater activations during the COPY condition (Figure 2A, RED regions, $Z>2.3$, cluster-corrected for multiple comparisons). Across the entire frontal lobe, there was a bias toward greater activation to COPY assignment in the right hemisphere in righthanded subjects. This was evident in the frontopolar cortex [FPC; Right hemisphere: Montreal Neuroscience Institute (MNI) coordinates $(32,58,0) \mathrm{mm}$; \% signal change $0.14 p<0.001$ Bonferroni corrected], the middle frontal gyrus [MNI coordinates $(44,18,38)$ $\mathrm{mm}$; signal change $0.11 ; p<0.01$ Bonferroni corrected] and the posterior precuneus $[\mathrm{MNI}$ coordinates $(4,-66,36) \mathrm{mm}$; $\%$ signal change $0.15 ; p<0.01$ Bonferroni corrected].

The degree of bias toward right hemisphere activation in righthanded subjects varied between individuals. Furthermore, examination of the responses in FPC for the two left-handed individuals in the study showed the largest bias in favor of the left frontal lobe. A formal test using the Laterality Index (Wilke and Lidzba, 2007) confirmed that there was a significant laterality shift toward right hemisphere activation of the frontal lobe in right-handed participants (Bootstrap method; Wilke and Schmithorst, 2006, $p<0.05$; two-tailed; Figure 2B). More data using this paradigm with more left-handed subjects would be needed to determine whether their frontopolar activations are lateralized in the left hemisphere.

There was also a modest increase in the activation of medial orbitofrontal cortex during the AUTHENTIC condition 


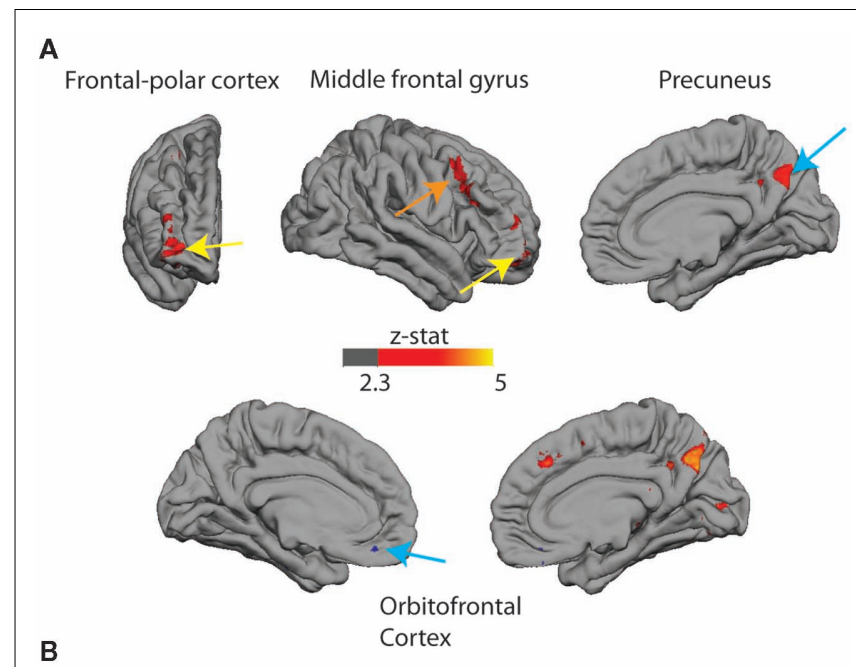

B

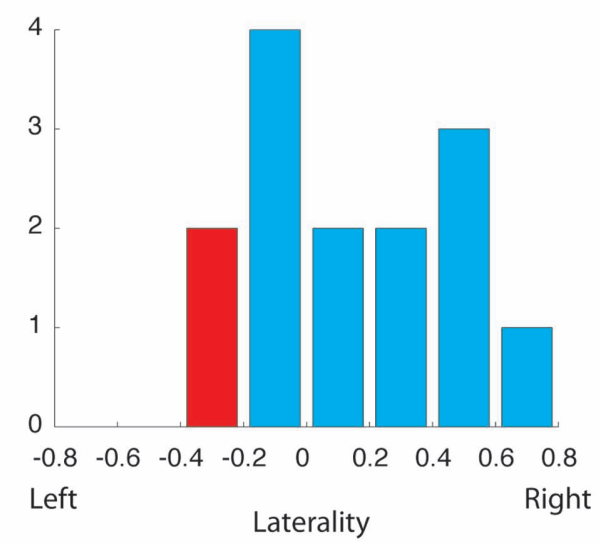

FIGURE 2 | (A) Activation ( $Z>2.3$, corrected) to the assignment of authenticity (AUTHENTIC vs. COPY). Upper: frontal, lateral, and medial views of right cortical hemispheres of right-handed participants, greater activation to COPY (red) in right frontopolar cortex (FPC; signal change $0.14 \%, p<0.001$, Bonferroni corrected); greater activation to COPY (red) in middle frontal gyrus (signal change $0.11 \%, p<0.01$, Bonferroni corrected); and greater activation to $\mathrm{COPY}$ (red) in right posterior precuneus (signal change $0.15 \%, p<0.01$, Bonferroni corrected). Lower: medial views of left and right cortex; greater activation to AUTHENTIC (blue) in medial orbitofrontal cortex: left, signal change $0.39 \%, p>0.05$; right, signal change $0.09 \%, p<0.01$, Bonferroni corrected; and greater activation to COPY (red) in right posterior precuneus. (B) Distribution of lateralization index (+1 right-sided, 0 balanced, -1 left-sided) for FPC activation for right-handed (BLUE) and left-handed (RED) participants.

(Figure 2A; BLUE regions, $Z>2.3$, uncorrected for multiple comparisons [Right hemisphere: MNI coordinates $(4,36,-22)$ $\mathrm{mm}$; \% signal change $0.39, p>0.05 \mathrm{NS}$. Left hemisphere: MNI coordinates $(-12,42,-16) \mathrm{mm}$; \% signal change $0.09, p<0.01$, Bonferroni corrected]. Inclusion of activations from all participants (right and left-handed) slightly improves the statistical power and confirms that the orbitofrontal activations appear to be bilateral.

PSYCHO-PHYSIOLOGICAL INTERACTION WITH THE OCCIPITAL CORTEX Although there was no difference in the response of visual cortex to COPY and AUTHENTIC assignments, further analysis indicated a highly specific link between the signals in FPC and those in the visual cortex. PPI analysis aims to investigate changes in the interaction between two brain areas under different psychological conditions (Friston et al., 1997). Since FPC showed activation to COPY > AUTHENTIC, this region is a potential source of top-down modulation of visual responses.

Figure 3A plots the responses of a voxel in the occipital cortex as a function of the responses of the peak-responding voxel in right FPC, separately for the COPY and AUTHENTIC assignments, for a single participant. The COPY condition induces a stronger correlation between the signals in the two brain regions. These correlated signals are evident in the group PPI for right-handed participants (Figure 3B), which showed significantly higher correlation of activity between the FPC ROI seed and several visual areas, including the LOC bilaterally $(Z=2.3, p<0.01$; corrected for multiple comparisons). A PPI is also found in visual cortex in association with activation of right precuneus although the extent of activation is greater in the left hemisphere (Figure 3B).

The PPI is identified within the context of a regression model with main effects (activation of each cortical area independently) and an interaction term (co-activation of two cortical areas, assessed by the correlation between activated voxels in the two areas), in which a statistical test is applied to test whether the co-activation is changed by the psychological condition (in our case, the assignment of authenticity). As such, the analysis cannot identify the direction of causality between the two cortical areas. We examined the time-course of activations in FPC and occipital cortex for evidence that the activations in one region preceded another in time. There was no clear evidence for such an effect, although the unambiguous identification of such differences in timing is often difficult (Smith et al., 2011).

Overall then, for our results, a significant statistical outcome is consistent with a top-down signal from FPC to occipital regions or a redirection of outputs from occipital regions away from other cortical regions toward FPC, with no net change in the activation of the occipital regions. A third, and in some ways most likely, possibility is that there is an increase in the cortical signaling that is passing in both directions between the two cortical regions (FPC and occipital).

\section{DISTINCTION OF REAL AND FAKE ARTWORKS}

Finally, we examined whether there were any differences in the cortical responses to the REAL and FAKE Rembrandts, regardless of the assignment of authenticity. The only small difference we found was confined to the neighborhood of the calcarine sulcus, which we attribute to small mismatches of contrast or visual symmetry between the chosen image sets (see Appendix, Figure A1).

\section{DISCUSSION}

Viewing of portrait art elicited the predicted activation in lateral visual cortical areas, corresponding to regions sensitive to faces and object recognition. However, these areas were not differentially activated by the cue of authenticity. Other areas were significantly activated by the assignment of authenticity, including the right FPC, right middle temporal gyrus, right precuneus, and orbitofrontal cortex.

The COPY assignment resulted in the stronger activations, in FPC and right posterior precuneus. To understand these outcomes, it is important to recall that, in response to the cue that the artwork 


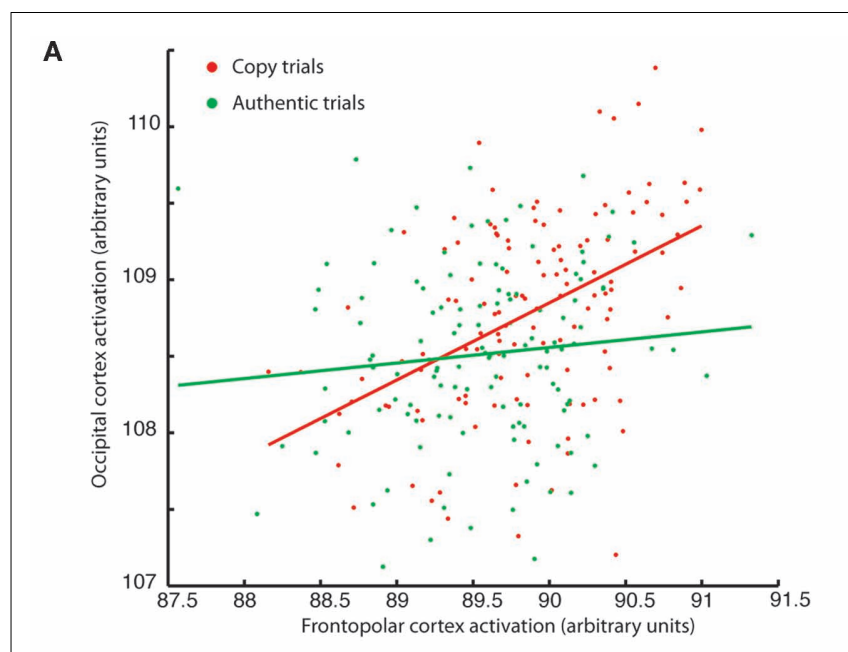

B

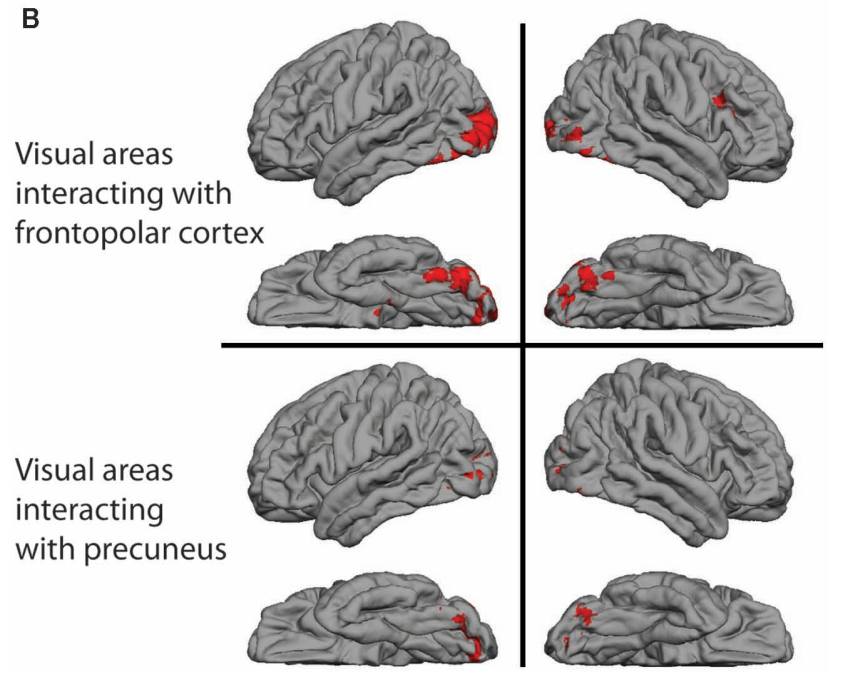

FIGURE 3 | Responses of right frontopolar cortex (FPC) and precuneus and their interaction with visual processing of images. (A) Functional activations, under COPY and AUTHENTIC assignments, of occipital regions of a single participant plotted against activations in right FPC. Stronger correlation between signals under COPY assignment $(r=0.46 ; p<0.0001)$ compared with AUTHENTIC $(r=0.11 ; p>0.2)$. (B) Psycho-physiological interaction (PPI) on group data from all right-handed participants, showing regions of occipital cortex whose correlation with right FPC (upper) and precuneus (lower) increases significantly in the COPY condition compared with the AUTHENTIC.

was a COPY, many participants reported that they were actively engaged in trying to detect the flaws in the presented image to gain confirmatory evidence in favor of the assignment. Participants also reported that their working hypothesis about what distinguished genuine and derivative works shifted over time as they viewed more images.

Activation of FPC (Brodmann area 10) has been obtained previously in studies that require information to be held in working memory: what is similar and relevant to our current study is that these are tasks in which multiple goals and hypotheses are being evaluated at the same time (Koechlin and Hyafil, 2007). Given that art experts combine multiple sources of information to make judgments about authenticity, the activations of FPC observed in this study are consistent with the idea that our participants are actively building hypotheses about the visual content of the images to determine which are genuine and which are not. The right middle temporal gyrus is often activated in working memory tasks, particularly those in which participants are processing spatial information (Leung et al., 2002).

The precuneus has been associated with many higher cognitive functions, including consciousness, aspects of memory and the experience of agency (Cavanna and Trimble, 2006), but it has been argued recently that this diversity of results may partly reflect a failure to identify clearly the functional compartments of this cortical region (Margulies et al., 2009). The activation seen in this study is clearly lateralized to the right and within the posterior zone closely connected with visual cortical areas. Given that participants reported that they were actively engaged in hypothesis-seeking about visual images, our findings are consistent with the proposal that the posterior precuneus forms part of a network with other cortical areas that are more purely visual (Dejong et al., 1994) in their responsiveness.

Both the PFC and precuneus show a PPI with regions of the occipital lobe: the distribution of occipital, cortical areas identified in the PPI (Figure 3B) is similar to the cortical activations generated by the paintings themselves (Figure 1). This result is particularly interesting as it suggests a greater functional interaction between an executive function in FPC and sensory signals in LOC, when images are cued as COPY. The only region activated by assignment of AUTHENTIC was the orbitofrontal cortex, which has been associated with reward and monetary gain (Gottfried et al., 2003; Gold and Shadlen, 2007; Padoa-Schioppa and Assad, 2008), presumably reflecting the increase in the perceived value of the artwork. Since this result is strongly expected based on current hypotheses about the function of orbitofrontal cortex, the presence of this significant activation gives important support to our experimental paradigm. Since the assignment of a portrait as AUTHENTIC enhances the perceived value in the eye of the viewer, we can conclude that the method of delivering advice to the participants in this study was effective and relevant.

The design of our study needed to balance the experience of free viewing typical during art appreciation and the detailed level of experimental control achieved in many neuroscientific studies. Viewing computer presentations of artwork whilst lying supine in the brain scanner is vastly different from viewing artwork in a gallery space, or even looking at reproductions in books in normal contexts. Equally, there was no specific task in relation to each image presentation and eye movements could roam freely across the image. These features allow some license in the cognitive strategies that individual participants might employ during this experiment. Nonetheless, the focus on artwork of a particular style in combination with a clear methodological question has yielded some striking results.

The brain areas, which we find are activated by assignment of authenticity, emphasize the cognitive element of viewing artwork. Authenticity is just one component of the viewer's experience during the appreciation of a work of art. Even so, manipulation of this individual element subtly modulates the interaction between multiple brain regions of the participants. It may be said that one 
of the tasks of a writer on art is to achieve such a modulation in the viewers' responses. Additionally, the production of new artwork is generally held to initiate a transaction between the artist and the prospective viewers, within a social and cultural context that is often fuzzy and soft-edged - and therefore very susceptible to direction. The brain interactions that we have identified form part of the way that humans respond in this social and cognitive setting.

\section{AUTHOR CONTRIBUTIONS}

The study was designed by Mengfei Huang, Martin J. Kemp, and Andrew J. Parker. Mengfei Huang implemented the design, collected initial results and conducted analysis under the supervision of Holly Bridge and Andrew J. Parker. Mengfei Huang wrote the initial working draft of the paper. Holly Bridge acquired data from more participants and undertook further analysis. Holly Bridge and Andrew J. Parker completed writing of the paper and all authors contributed to design and presentation of figures.

\section{ACKNOWLEDGMENTS}

This work was supported by grants from the Wellcome Trust and James S. McDonnell Foundation to Andrew J. Parker, by a Royal Society University Research Fellowship to Holly Bridge and a Fulbright award to Mengfei Huang.

\section{REFERENCES}

Asch, S. E. (1956). Studies of independence and conformity: a minority of one against a unanimous majority. Psychol. Monogr. 70. (9, Whole No. 416).

Bossart, W. (1961). Autheniticity and Aesthetic value in the visual arts. $B r$. J. Aesthetics 1, 144-159.

Cavanna, A. E., and Trimble, M. R. (2006). The precuneus: a review of its functional anatomy and behavioural correlates. Brain 129, 564-583.

Dejong, B. M., Shipp, S., Skidmore, B., Frackowiak, R. S. J., and Zeki, S. (1994). The cerebral-activity related to the visual-perception of forward motion in-depth. Brain 117, 1039-1054.

Friston, K. J., Buechel, C., Fink, G. R., Morris, J., Rolls, E., and Dolan, R. J. (1997). Psychophysiological and modulatory interactions in neuroimaging. Neuroimage 6, 218-229.

Gold, J. I., and Shadlen, M. N. (2007). The neural basis of decision making. Annu. Rev. Neurosci. 30, 535-574.

Goodman, N. (1976). "Art and Authenticity," in Languages of Art. Indianapolis: Hackett Publishing Co, 99-123.

Gottfried, J. A., O'doherty, J., and Dolan, R. J. (2003). Encoding predictive reward value in human amygdala and orbitofrontal cortex. Science 301, 1104-1107.

Grill-Spector, K., Kushnir, T., Edelman, S., Avidan, G., Itzchak, Y., and Malach, R. (1999). Differential processing of objects under various viewing conditions in the human lateral occipital complex. Neuron 24, 187-203.

Ishai, A., Ungerleider, L. G., Martin, A., and Haxby, J. V. (2000). The representation of objects in the human occipital and temporal cortex. J. Cogn. Neurosci. 12, 35-51.
Jenkinson, M., Bannister, P., Brady, M., and Smith, S. (2002). Improved optimization for the robust and accurate linear registration and motion correction of brain images. Neuroimage 17, 825-841.

Jenkinson, M., and Smith, S. (2001). A global optimisation method for robust affine registration of brain images. Med. Image Anal. 5, 143-156.

Kanwisher, N., Mcdermott, J., and Chun, M. M. (1997). The fusiform face area: a module in human extrastriate cortex specialized for face perception. J. Neurosci. 17, 4302-4311.

Kastner, S., Pinsk, M. A., De Weerd, P., Desimone, R., and Ungerleider, L. G. (1999). Increased activity in human visual cortex during directed attention in the absence of visual stimulation. Neuron 22, 751-761.

Kastner, S., and Ungerleider, L. G. (2000). Mechanisms of visual attention in the human cortex. Annu. Rev. Neurosci. 23, 315-341.

Kawabata, H., and Zeki, S. (2004). Neural correlates of beauty. J. Neurophysiol. 91, 1699-1705.

Kawabata, H., and Zeki, S. (2008). The neural correlates of desire. PLoS ONE 3, e3027. doi:10.1371/journal.pone.0003027

Koechlin, E., and Hyafil, A. (2007). Anterior prefrontal function and the limits of human decision-making. Science 318, 594-598.

Krawczyk, D. C., Gazzaley, A., and D'Esposito, M. (2007). Reward modulation of prefrontal and visual association cortex during an incentive working memory task. Brain Res. 1141, 168-177.

Leung, H. C., Gore, J. C., and Goldman-Rakic, P. S. (2002). Sustained mnemonic response in the human middle frontal gyrus during on-line storage of spatial memoranda. J. Cogn. Neurosci. 14, 659-671.
Margulies, D. S., Vincent, J. L., Kelly, C., Lohmann, G., Uddin, L. Q., Biswal, B. B., Villringer, A., Castellanos, F. X., Milham, M. P., and Petrides, M. (2009). Precuneus shares intrinsic functional architecture in humans and monkeys. Proc. Natl. Acad. Sci. U.S.A. 106, 20069-20074.

McClure, S. M., Li, J., Tomlin, D., Cypert, K. S., Montague, L. M., and Montague, P. R. (2004). Neural correlates of behavioral preference for culturally familiar drinks. Neuron 44, 379-387.

Mojzisch, A., and Krug, K. (2008). Cells, circuits, and choices: social influences on perceptual decision making. Cogn. Affect. Behav. Neurosci. 8, 498-508.

Offen, S., Schuppeck, D., and Heeger, D. J. (2009). The role of early visual cortex in visual short-term memory and visual attention. Vision Res. 49, 1352-1362.

Padoa-Schioppa, C., and Assad, J. A. (2008). The representation of economic value in the orbitofrontal cortex is invariant for changes of menu. Nat. Neurosci. 11, 95-102.

Prins, N. (2007). Phase Spectrum Scrambling. Available: http://visionscience.com/pipermail/ visionlist/2007/002181.html

Seinstra, F. J. (2010). A Web Catalogue of Rembrandt Paintings. Amsterdam: University of Amsterdam.

Shuler, M. G., and Bear, M. F. (2006). Reward timing in the primary visual cortex. Science 311, 1606-1609.

Smith, J., and Smith, L. (2001). Spending time on art. Empir. Stud. Arts 19, 229-236.

Smith, S. (2002). Fast robust automated brain extraction. Hum. Brain Mapp. 17, 143-155.

Smith, S. M., Jenkinson, M., Woolrich, M. W., Beckmann, C. F., Behrens, T. E. J., Johansen-Berg, H., Bannister, P. R., De Luca, M., Drob- njak, I., Flitney, D. E., Niazy, R. K., Saunders, J., Vickers, J., Zhang, Y. Y., De Stefano, N., Brady, J. M., and Matthews, P. M. (2004) Advances in functional and structural MR image analysis and implementation as FSL. Neuroimage 23, S208-S219.

Smith, S. M., Miller, K. L., SalimiKhorshidi, G., Webster, M., Beckmann, C. F., Nichols, T. E., Ramsey, J. D., and Woolrich, M. W. (2011). Network modelling methods for FMRI. Neuroimage 54, 875-891.

von Sonnenburg, H., Liedtke, W., Logan, C., Orenstein, N., and Dickey, S. (1995). "Rembrandt/Not Rembrandt in the Metropolitan Museum of Art," in Exhibition Catalogue, Vol. 1 and 2, New York, NY: Metropolitan Museum of Art.

Wilke, M., and Lidzba, K. (2007). LI-tool: a new toolbox to assess lateralization in functional MRdata. J. Neurosci. Methods 163, 128-136.

Wilke, M., and Schmithorst, V. J. (2006). A combined bootstrap/histogram analysis approach for computing a lateralization index from neuroimaging data. Neuroimage 33, 522-530.

Woolrich, M. W., Jbabdi, S., Patenaude, B., Chappell, M., Makni, S., Behrens, T., Beckmann, C., Jenkinson, M., and Smith, S. M. (2009). Bayesian analysis of neuroimaging data in FSL. Neuroimage 45, S173-S186.

Woolrich, M. W., Ripley, B. D., Brady, M., and Smith, S. M. (2001). Temporal autocorrelation in univariate linear modeling of FMRI data. Neuroimage 14, 1370-1386.

Wynne, F. (2006). I Was Vermeer. London: Bloomsbury.

Zaksas, D., and Pasternak, T. (2006). Directional signals in the prefrontal cortex and in area MT during a working memory for visual motion task. J. Neurosci. 26, 11726-11742. 
Conflict of Interest Statement: The authors declare that the research was conducted in the absence of any commercial or financial relationships that could be construed as a potential conflict of interest.
Received: 07 April 2011; accepted: 24 October 2011; published online: 28 November 2011.

Citation: Huang $M$, Bridge H, Kemp MJ and Parker AJ (2011) Human cortical activity evoked by the assignment of authenticity when viewing works of art. Front. Hum. Neurosci. 5:134. doi: 10.3389/fnhum.2011.00134

Copyright (C) 2011 Huang, Bridge, Kemp and Parker. This is an open-access article subject to a non-exclusive license between the authors and Frontiers Media $S A$, which permits use, distribution and reproduction in other forums, provided the original authors and source are credited and other Frontiers conditions are complied with. 


\section{APPENDIX \\ BRAIN RESPONSES TO REAL AND FAKE REMBRANDT PORTRAITS}

The focus of the main study was the brain's response to assignment of authenticity rather than authenticity of the artworks depicted by the images. Nonetheless, the design of the experimental protocol makes it possible to use the same data set to determine whether the brain responds differently to images of REAL and FAKE Rembrandt portraits. The analysis methodology was identical to that in the main paper, except that in this case the contrast visualREAL $>$ visualFAKE is of primary interest. The outcome is shown in Figure A1. This shows that all significant differences in REAL and FAKE portraits are confined to early visual areas in the close neighborhood of the calcarine sulcus (marked CS in Figure). The pattern of response is such that the REAL images generate stronger responses in left visual cortex and FAKE images generate stronger responses in right visual cortex. Almost certainly, this does not indicate a lateralized brain response to REAL and FAKE images. The most probable cause is a small mismatch in the distribution of small image features or contrast in the two sets of images, which our selection procedure had failed to eliminate. Although the images were carefully chosen to balance the numbers of left and right three-quarter views, it is nonetheless all too easy for some small bias of this kind to remain. This interpretation is consistent with the absence of activations anywhere else in the brain and most particularly, the absence of activations for visualREAL $>$ visualFAKE that correspond in cortical location to the activations to visualAUTHENTIC $>$ visualCOPY presented in the main paper. We also examined the data set for interactions between the AUTHENTIC > COPY and REAL > FAKE contrasts but nothing additional emerged.

\section{SCREENING OUESTIONNAIRE}

The following questionnaire was applied to all potential participants before recruiting them to ensure that all participants were amateur viewers (visit art museums 5 times/year; no extensive art training). The process also screened out individuals who dislike Rembrandt. To avoid potentially confounding variables. Most importantly, it confirmed their familiarity with Rembrandt's fame among artists, with all participants ranking him among the top 25 artists of all time, or higher

1. How often do you visit art exhibitions, galleries, or museums?
a. 0 times/year
b. 1-4 times/year
c. 5-10 times/year
d. 11-15 times/year
e. 16-20 times/year

2. Have you heard of the artist Rembrandt? $(\mathrm{Y} / \mathrm{N})$

3. How familiar are you with the work of Rembrandt? (on a scale of 1-5)

1. Never seen any of his work

2. Somewhat familiar

3. Familiar

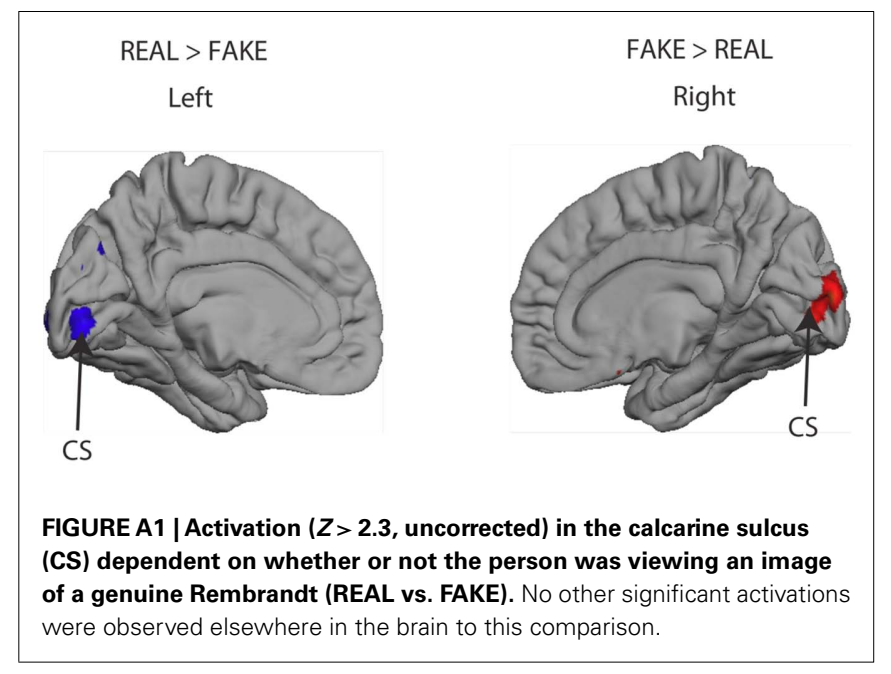

4. Very familiar

5. Extremely familiar

4. How much do you like the work of Rembrandt? (on a scale of $1-5)$

$1=$ strongly dislike

$2=$ somewhat dislike

$3=$ neutral

$4=$ somewhat like

$5=$ strongly like

5. Would you consider Rembrandt to be one of...
a. the top 5 most famous artists of all times
b. the top 10
c. the top 25
d. the top 50

6. Do you have any visual arts-related interests, hobbies, or skills? (Please specify the duration of involvement).

\section{POST-SCAN QUESTIONNAIRE}

The following questionnaire was applied to all participants immediately after their session in the MRI scanner. The verbal responses were used to check that participants had remained alert and active during the scan and to highlight subjective aspects of the participants' experiences.

1. How was it? Did you fall asleep or blank out at any point? (Opportunity to let them make any comments, and for PI to be aware of any specific circumstances).

2. What did you think of the authentic paintings vs. the copies? Any differences?

3. Did you have a particular approach or strategy for looking at each image? (e.g., some people start with the face, some focus on the colors or the lighting).

4. What were you thinking about during the breaks between each image? 